\title{
Chronology of Magnetic Reversals
}

THERE is a certain monumental quality about the geomagnetic survey of the lavas of Eastern Iceland reported on page 25 of this issue. Although the long succession of overlapping lava flows in Iceland has played an important part in the development of rock magnetism in the past fifteen years, the study which has now been carried out is at once more detailed and more comprehensive than most others. Altogether there are some 900 separate lava flows lying on top of each other and these have been sampled at a score of places by cores drilled to the underlying basalt. The direction of magnetization of more than 2,000 samples representative of individual lava flows has been determined. Perhaps the most valuable of the prizes to be won by this activity is an extension of the chronology of the sequence of magnetic field reversals from a few million years to an interval of time which spans the past twenty million years. Iceland is exceptionally well suited to such an enterprise because of its close geological relationship with the mid-Atlantic Ridge.

So far as can be told, chronology for the sequence of reversals of the Earth's magnetic field obtained from Eastern Iceland agrees well with other studies which span a shorter interval of time. Precision in this kind of work is possible only when direct measurements can be made of the age of rocks found to be polarized in a direction opposite to that of the contemporary magnetic field of the Earth. Potassium-argon dating has been of crucial importance in this respect. A year ago, Doell and Dalrymple were able, for example, to fix the age of a sequence of volcanic rocks in New Mexico laid down between 700,000 and one million years ago, which placed the most recent reversal of the Earth's magnetic field at 700,000 years ago. The same measurements would suggest that that period of reversed magnetism was comparatively short lived100,000 years or so. It seems as if the youngest of the lavas in the sequence from Eastern Iceland, corresponding as it does to a period of normal magnetization, coincides either with the period of normal magnetization just this side of a million years ago or that immediately before it. It is, however, more striking that the long sequence of sixty changes of polarity now identified in Iceland agrees both in character androughly--in chronology with the sequence of magnetic reversals which appears to be fossilized in the igneous rocks spreading outwards from the ocean ridges. On the assumption that the rate of spreading of the ocean floor is a centimetre a year or thereabouts, the rocks 100 kilometres each side of an ocean ridge span a total of ten million years. As Dagley and his collaborators point out, it is a pleasing feature of their work that 32 reversals of the Earth's magnetic field ago, they find a long period of normal magnetization which coincides with such a one already reported from studies of the ocean floor near the East Pacific Rise. Plainly there is at least a possibility that further work along these lines, and the patient correlation of measurements with rocks which can be dated easily and those whose ages must be inferred from assumptions about the rate of spreading of the ocean floor, will yield such a detailed picture of the pattern of reversals in the past 20 million years for the Earth's magnetism to be a rapid and an important means of dating all kinds of the Earth's physical phenomena. This, no doubt, is a goal which will be vigorously pursued.

One particularly interesting feature of the results of the survey in Iceland is that there appear to have been 73 lava flows whose directions of magnetization were anomalous and that at least 55 of these are unambiguously intermediate-magnetized neither in the normal direction nor the opposite direction. The fact that such a high proportion-five per cent or so-of the lava flows examined turn out to have anomalous polarity is itself a proof of how conspicuous a part of the history of the last twenty million years have been these processes of field reversal. With such a large number of examples with which to work, it seems fair to point out, as Dagley and his collaborators do, that this may be evidence for a comparatively gradual process of reversal. Moreover, it may indicate that in the course of a reversal, there is a swinging round of the direction of the Earth's magnetic field and not simply a diminution of its intensity and then an increase in the opposite direction. Whether evidence of the kind which has now been collected in Iceland will in itself be sufficient to decide unambiguously between several possibilities in this field remains to be seen, but there can be no doubt that a long sequence of measurements like those which have now been obtained will be of the greatest value in suggesting which hypotheses it will be most profitable to test.

\section{What Controls Chromosomes?}

The chromosomes of wheat, like those of Drosophila, have been an extraordinarily fruitful field of study. The reconstruction of the evolution of modern cereals in prehistory from the cytogenetical evidence is, of course, a classic; the Neolithic peoples of the Mediterranean were not geneticists, but it is clear that they were plant breeders with a vengeance. But the chromosomes of wheat have also been an important object study in the understanding of the functioning of chromosomes as such. So much is clear, yet again, from the report by Riley and Chapman (page 60, this issue) of the role of a particular chromosome of common wheat (Triticum aestivum) in the genetic control of the behaviour of chromosomes during the meiosis. The point at issue is 